\title{
ON HARDY'S INEQUALITY AND LAPLACE TRANSFORMS IN WEIGHTED REARRANGEMENT INVARIANT SPACES
}

\author{
KENNETH F. ANDERSEN ${ }^{1}$
}

ABSTRACT. Hardy's well-known inequality relating the norm of a function and the norm of its integral mean in the Lebesgue spaces $L^{p}(\mu), d \mu(t)=t^{\sigma-1} d t$, is extended to the class of rearrangement invariant spaces $X(\mu)$. These spaces include, for example, the $L^{p}(\mu)$, the Lorentz and the Orlicz spaces. As an application, necessary and sufficient conditions are obtained for an operator related to the Laplace transform to be bounded as a linear operator between rearrangement invariant spaces of $\mu$-measurable functions.

For $\sigma>0$, we write $d \mu(t)=t^{\sigma-1} d t$ and denote by $L^{p}(\mu)$ the space of $\mu$ measurable functions on $(0, \infty)$ for which the norm

$$
\|f\|_{p, \mu}= \begin{cases}\left(\int_{0}^{\infty}|f(t)|^{p} d \mu(t)\right)^{1 / p}, & 1 \leqq p<\infty, \\ \underset{t>0}{\operatorname{ess} \sup }|f(t)|, & p=\infty,\end{cases}
$$

is finite, and if $X$ and $Y$ are Banach spaces, $[X, Y]$ will denote the space of bounded linear operators from $X$ into $Y$. We abbreviate $[X, X]=[X]$. Let the operators $P$ and $P^{\prime}$ be defined by

$$
(P f)(s)=\frac{1}{s} \int_{0}^{s} f(t) d t \text { and }\left(P^{\prime} f\right)(s)=\int_{s}^{\infty} f(t) \frac{d t}{t}
$$

whenever the required integrals exist for all $s>0$. Then Hardy's celebrated theorem [4, pp. 245-246] may be stated in the form:

Theorem Suppose $1 \leqq p<\infty$. Then $P \in\left[L^{p}(\mu)\right]$ if $p>\sigma$ and $P^{\prime} \in$ $\left[L^{p}(\mu)\right]$ if $\sigma>0$.

In this paper we determine necessary and sufficient conditions which allow the $L^{p}(\mu)$ spaces which appear in Hardy's theorem to be replaced by a function space of a more general class. The class of spaces with which we deal possess the property of rearrangement invariance and include, for

Received by the editors July 6, 1972 and, in revised form, October 30, 1972.

AMS (MOS) subject classifications (1970). Primary 26A86, 44A10, 46E30.

Key words and phrases. Integral mean, Laplace transform, rearrangement invariant function space, Lorentz space, Orlicz space.

${ }^{1}$ Research supported in part by NRC grant \#A8185.

(c) American Mathematical Society 1973 
example, the $L^{p}(\mu)$ spaces, the Lorentz and the Orlicz spaces. We apply our results to the Laplace transform considered as a linear operator between rearrangement invariant spaces.

Following the approach of [2] we assume that $X(m), m$ denoting Lebesgue measure on $(0, \infty)$, is a Banach space of Lebesgue measurable functions on $(0, \infty)$ whose norm $\|\cdot\|_{X(m)}$ is rearrangement invariant in the sense that two functions which are equimeasurable with respect to $m$ have the same norm. The rearrangement invariant space $X(\mu)$ then consists of those $\mu$-measurable functions on $(0, \infty)$ for which $f^{*} \in X(m)$ and the norm in $X(\mu)$ is given by $\|f\|_{X(\mu)}=\left\|f^{*}\right\|_{X(m)}$. Here, as usual, $f^{*}$ denotes the nonnegative, nonincreasing rearrangement of $f$ which is equimeasurable with $f$ in the sense that

$$
\mu\{t:|f(t)|>y\}=m\left\{t: f^{*}(t)>y\right\} \quad(y>0) .
$$

For more details see [1], [2].

The upper index $\alpha$ and the lower index $\beta$ corresponding to the rearrangement invariant space $X$ was defined by Boyd [2] in terms of the function $h(s, X, X)$, where for rearrangement invariant spaces $X$ and $Y, h(s, X, Y)$ denotes the norm in $[X(m), Y(m)]$ of the dilation operator $E_{s}:\left(E_{s} f\right)(t)=$ $f(s t)$. Note that if $X \subseteq Y$, then $h(1, X, Y)$ is finite. This follows from the closed graph theorem and [1, Definition 1.1(iv)].

We have the following:

THEOREM 1. Let $X$ be a rearrangement invariant space with upper index $\alpha$ and lower index $\beta$. Then

(i) $P \in[X(\mu)]$ if and only if $\alpha \sigma<1$.

(ii) $P^{\prime} \in[X(\mu)]$ if and only if $\beta>0$.

THEOREM 2. Let $X$ and $Y$ be rearrangement invariant spaces and let $h(s)=h(s, X, Y)$. Then

(i) The condition $X \subseteq Y$ is necessary for $P \in[X(\mu), Y(\mu)]$ and the condition $\int_{0}^{1} h\left(s^{\sigma}\right) d s<\infty$ is sufficient for $P \in[X(\mu), Y(\mu)]$.

(ii) The condition $X \subseteq Y$ is necessary for $P^{\prime} \in[X(\mu), Y(\mu)]$ and the condition $\int_{1}^{\infty} h\left(s^{\sigma}\right) d s / s<\infty$ is sufficient for $P^{\prime} \in[X(\mu), Y(\mu)]$.

For the Lorentz space $L^{p, q}$ the indices are given by $\alpha=\beta=1 / p$, so in particular, taking $p=q$ in Theorem 1 we recover Hardy's theorem. The indices for the various Lorentz spaces $\Lambda(\varphi, p), M(\varphi, p)$ and the Orlicz spaces $L_{M \Phi}, L_{\Phi}$ have been computed by Boyd [1]. We leave to the reader the application of those results to our Theorem 1.

For the particular case $\sigma=1$, Theorems 1 and 2 were obtained by Boyd [1] and applied to a study of the Hilbert transform. Here, we give one application of our results to a transform which is related to the Laplace 
transform $\mathscr{L}$. Let the transform $T$ be given by

$$
(T f)(s)=\frac{1}{s}(\mathscr{L} f)\left(\frac{1}{s}\right)=\int_{0}^{\infty} e^{-t / s} f(t) \frac{d t}{s} \quad(s>0) .
$$

It is well known that $T \in\left[L^{p}(\mu)\right]$ if and only if $p>\sigma$, indeed, the case $\sigma=1$ is suggested in [5, p. 397, Ex. 16] as an application of Hardy's theorem. We prove the following:

THEOREM 3. Let $X$ and $Y$ be rearrangement invariant spaces. Then $T \in[X(\mu), Y(\mu)]$ if and only if $P \in[X(\mu), Y(\mu)]$.

COROLlaRY 1. If $X$ has upper index $\alpha$, then $T \in[X(\mu)]$ if and only if $\alpha \sigma<1$.

COROLlaRY 2. $X \subseteq Y$ is a necessary condition and $\int_{0}^{1} h\left(s^{\sigma}, X, Y\right) d s<\infty$ is sufficient for $T \in[X(\mu), Y(\mu)]$.

COROLlary 3. If $\sigma<1$, then $T \in[X(\mu), Y(\mu)]$ if and only if $X \subseteq Y$.

Corollaries 1 and 2 follow immediately from the theorems, and according to [1, p. 605 , Lemma 3.2] $s h(s, X, Y) \leqq h(1, X, Y)$ for $0<s<1$, so if $\sigma<1$ and $X \subseteq Y$ we have

$$
\int_{0}^{1} h\left(s^{\sigma}, X, Y\right) d s \leqq h(1, X, Y) \int_{0}^{1} s^{-\sigma} d s<\infty
$$

and Corollary 3 then follows from Corollary 2.

The theorems depend on the following lemma which is adapted from $\S 3$ of [1] and which deals with operators of the following form: Let $a(t)$ be nonnegative and measurable on $(0, \infty)$. Define

and

$$
(K f)(s)=\int_{0}^{\infty} a(t) f(s t) d t
$$

$$
(\tilde{K} f)(s)=\int_{0}^{\infty} \frac{1}{\sigma} t^{1 / \sigma} a\left(t^{1 / \sigma}\right) f(s t) \frac{d t}{t}
$$

whenever the required integrals exist for all $s>0$.

LEMmA. Suppose $X$ and $Y$ are rearrangement invariant spaces and $K, \tilde{K}$ are as defined above.

(a) $K \in[X(\mu), Y(\mu)]$ if and only if $\widetilde{K} \in[X(m), Y(m)]$, indeed, $K$ and $\tilde{K}$ have the same norm in the respective spaces.

(b) If $c=\int_{0}^{\infty} a(s) h\left(s^{\sigma}, X, Y\right) d s<\infty$, then $K \in[X(\mu), Y(\mu)]$ with $\|K\| \leqq c$.

(c) If $K \in[X(\mu), Y(\mu)]$ and $A(s)=\int_{0}^{s} a(t) d t$ then $A(s) h\left(s^{\sigma}, X, Y\right) \leqq$ $\|K\|$. 
(d) If $a(t)>0$ on a set of positive measure and $K \in[X(\mu), Y(\mu)]$ then $X \subseteq Y$.

Proof. Let $f \in X(m)$ and define, for $t>0, g(t)=(\tau f)(t)=f\left(t^{\sigma} / \sigma\right)$. Then, for each $y>0$,

$$
\begin{aligned}
m\left\{t: g^{*}(t)>y\right\} & =\mu\{t:|g(t)|>y\} \\
& =\mu\left\{t:\left|f\left(t^{\sigma} / \sigma\right)\right|>y\right\}=m\{t:|f(t)|>y\}
\end{aligned}
$$

so that for any rearrangement invariant space $Z$ we have

$$
\|\tau f\|_{Z(\mu)}=\|g\|_{Z(\mu)}=\left\|g^{*}\right\|_{Z(m)}=\|f\|_{Z(m)} .
$$

Now,

$$
\begin{aligned}
(K g)\left((\sigma s)^{1 / \sigma}\right) & =\int_{0}^{\infty} a(t) g\left((\sigma s)^{1 / \sigma} t\right) d t=\int_{0}^{\infty} a(t) f\left(s t^{\sigma}\right) d t \\
& =\int_{0}^{\infty} \frac{1}{\sigma} t^{1 / \sigma} a\left(t^{1 / \sigma}\right) f(s t) \frac{d t}{t}=(\tilde{K} f)(s)
\end{aligned}
$$

that is, $K(\tau f)=\tau(\tilde{K} f)$ and hence, from (1),

$$
\|\tilde{K} f\|_{Y(m)}=\|\tau(\tilde{K} f)\|_{Y(\mu)}=\|K(\tau f)\|_{X(\mu)}
$$

from which (a) follows. Now according to [1, Theorem 3.1], $\tilde{K} \in[X(m)$, $Y(m)$ ] whenever

$$
\int_{0}^{\infty} a(s) h\left(s^{\sigma}, X, Y\right) d s=\int_{0}^{\infty} \frac{1}{\sigma} s^{1 / \sigma} a\left(s^{1 / \sigma}\right) h(s, X, Y) \frac{d s}{s}<\infty,
$$

so (b) follows from (a). Finally, (c) and (d) follow easily from (a) and [1, Lemma 3.3].

Note that the operators $P, P^{\prime}$, and $T$ are, for appropriate choices of $a(t)$, of the form $K$ in the lemma. In particular, Theorem 2 follows immediately from the lemma, and we now prove Theorems 1 and 3.

Proof of Theorem 1. According to (a) of the lemma, $P \in[X(\mu)]$ if and only if $\tilde{P} \in[X(m)]$ where

$$
(\widetilde{P} f)(s)=\int_{0}^{1} \frac{1}{\sigma} t^{1 / \sigma} f(s t) \frac{d t}{t},
$$

so (i) follows from (50) of [2]. Again by the lemma, $P^{\prime} \in[X(\mu)]$ if and only if $P^{\prime}=\sigma \widetilde{P}^{\prime} \in[X(m)]$. Now if $X^{\prime}$ is the associate space of $X$ with upper index $\alpha^{\prime}$, then $P^{\prime} \in[X(m)]$ if and only if $P \in\left[X^{\prime}(m)\right]$, and since $\beta=1-\alpha^{\prime}$, (ii) follows from (i).

Proof of Theorem 3. Since

$$
(P|f|)(s)=\int_{0}^{1}|f(s t)| d t \leqq e \int_{0}^{\infty} e^{-t}|f(s t)| d t=e(T|f|)(s)
$$


we get $P \in[X(\mu), Y(\mu)]$ whenever $T \in[X(\mu), Y(\mu)]$. On the other hand, $T|f| \leqq P|f|+Q|f|$ where

$$
(Q f)(s)=\int_{1}^{\infty} e^{-t} f(s t) d t \quad(s>0),
$$

so we need only show that $Q \in[X(\mu), Y(\mu)]$ whenever $P \in[X(\mu), Y(\mu)]$. Now if $P \in[X(\mu), Y(\mu)]$ then $X \subseteq Y$ by Theorem 2 so that

$$
\int_{1}^{\infty} e^{-s} h\left(s^{\sigma}, X, Y\right) d s \leqq h(1, X, Y) \int_{1}^{\infty} e^{-s} d s<\infty
$$

and $Q \in[X(\mu), Y(\mu)]$ by (b) of the lemma.

\section{REFERENCES}

1. D. W. Boyd, The Hilbert transformation on rearrangement invariant spaces, Canad. J. Math. 19 (1967), 599-616. MR 35 \#3383.

2. - Indices of function spaces and their relationship to interpolation, Canad. J. Math. 21 (1969), 1245-1254.

3. - Indices for the Orlicz spaces, Pacific J. Math. 38 (1971), 315-323.

4. G. H. Hardy, J. E. Littlewood and G. Pólya, Inequalities, Cambridge Univ. Press, New York, 1934.

5. E. C. Titchmarsh, The theory of functions, 2nd ed., Clarendon Press, Oxford, 1948.

Department of Mathematics, University of Alberta, Edmonton, Alberta, Canada 